\title{
Síndrome de Sweet y enfermedad inflamatoria intestinal. Aportación de un nuevo caso y revisión de la literatura
}

\author{
J. C. ANGLADA PINTADO, A. MICHÁN DOÑA, A. SILVA ABAD*, A. ZAPATA \\ LÓPEZ, S. ALCARAZ GARCÍA, P. RIANDE GONZÁLEZ**, E. MENOR CAMPOS \\ Hospital del S.A.S. de Jerez de la Frontera. Sección de Medicina Interna, *Anatomía \\ Patológica y **Dermatología. Jerez de la Frontera. Cádiz
}

SWEET'S SYNDROME AND INTESTINAL INFLAMMATORY DISEASE. A CASE REPORT AND REVIEW OF THE LITERATURE

\begin{abstract}
RESUMEN
El síndrome de Sweet o dermatosis neutrofílica aguda febril es un proceso caracterizado por fiebre, placas eritematosas dolorosas, neutrofilia y un infiltrado dérmico constituido por neutrófilos con edema papilar sin vasculitis. Su asociación con la enfermedad inflamatoria intestinal es inusual, presentamos un nuevo caso de síndrome de Sweet asociado a colitis ulcerosa, y realizamos una revisión de los casos descritos en la literatura asociados a enfermedad inflamatoria intestinal.
\end{abstract}

PALABRAS CLAVE: Enfermedad inflamatoria intestinal. Síndrome de Sweet. Colitis ulcerosa. Enfermedad de Crohn.

\begin{abstract}
Sweet's syndrome, or febrile neutrophilic dermatosis, is a process characterized by fever, red tender plaques, neutrophilia and neutrophilic dermal infiltrate with papilar edema in the absence of vasculitis. The association with intestinal inflammatory disease is inusual, a new case associated with ulcerative colitis is reported, and we perform a literature review of Sweet's associated intestinal inflammatory disease.
\end{abstract}

KEY WORDS: Inflammatory bowel disease. Sweet's syndrome. Ulcera tive colitis. Crohn's disease.

Anglada Pintado JC, Michán Doña A, Silva Abad A, Zapata López A, Alcaraz García S, Riande González P, Menor Campos E. Síndrome de Sweet y enfermedad inflamatoria intestinal. Aportación de un nuevo caso y revisión de la literatura. An Med Interna (Madrid) $2002 ; 19: 419-422$.

\section{INTRODUCCIÓN}

El síndrome de Sweet (SS) o dermatosis neutrofílica aguda febril, es una entidad clínica descrita por primera vez en 1964 por Robert Douglas Sweet. Se caracteriza por la aparición súbita de placas eritematosas y dolorosas que se localizan predominantemente en brazos, zona más alta del tronco, cuello y cara. A esto se asocia generalmente deterioro del estado general, con leucocitosis, neutrofilia y fiebre. Los hallazgos histológicos típicamente demuestran un marcado edema con un infiltrado dérmico predominantemente neutrofílico en ausencia de vasculitis leucocitoclástica (1). Su y Han-Nan (2) han propuesto una serie de criterios diagnósticos que se encuentran resumidos en la tabla I. En la actualidad se desconoce su etiología, se cree que es debido a un fenómeno de hipersensibilidad a varios antígenos desconocidos, puede ser idiopático aunque hasta en un $50 \%$ de los casos existe una enfermedad subyacente (3). Se describen múltiples asociaciones, en el $10 \%$ coexiste con una enfermedad hematológica maligna, principalmente leucemia mieloide aguda (4), aunque también se asocia a leucemia mieloide crónica, mielodisplasia, síndromes mieloproliferativos y tumores de células T y B (5). Se ha descrito asociado a carcinoma de ovario (6), carcinoma de esófago (7), síndrome de Reiter (8), síndrome de Sjögren (9), artritis reumatoide (10), enfermedad de Behçet (11), psoriasis (12), síndrome de Dressler (13), infección por el virus de la inmunodeficiencia humana (4) y a la enfermedad intestinal inflamatoria (1).

Aportamos a continuación un nuevo caso asociado a colitis ulcerosa $(\mathrm{CU})$ y realizamos la revisión de los casos descritos en la literatura (MEDLINE hasta Mayo de 2000). Analizamos la edad, sexo, distribución y actividad de la enfermedad inflamatoria intestinal, manifestaciones acompañantes, relación temporal entre la aparición del SS y el proceso intestinal inflamatorio.

\section{CASO APORTADO}

Mujer de 58 años de edad, entre cuyos antecedentes personales destacaban obesidad y diabetes mellitus tipo 2 insulinrequiriente, diagnosticada en Junio del 1998 ( 1 año antes de su ingreso) de colitis ulcerosa con afectación de colon y recto. Seguía tratamiento con olsalazina y esteroides que fueron suprimidos en Marzo de 1999. A mediados de Abril comienza con lesiones dérmicas eritematosas y

Trabajo aceptado: 05 de febrero de 2001

Correspondencia: Juan Carlos Anglada Pintado. Sección de Medicina Interna. Hospital del S.A.S. de Jerez. Carretera de Circunvalación, s/n. 11407 Jerez de la Frontera. Cádiz.e-mail: jcanglada@yahoo.es 


\section{TABLA I}

\section{CRITERIOS DIAGNÓSTICOS DEL SÍNDRO ME DE SWEET}

Criterios mayores
A parición súbita de placas o nódulos eritematosos o vio-
láceos y dolorosos
Infiltración dérmica de polimorfonucleares neutrófilos sin
vasculitis leucocitoclástica

\section{Criterios menores}

\section{Prodrómos ded fiebre o de proceso infeccioso}

\section{Leucocitosis}

\section{Asociación de artralgias, conjuntivitis, fiebre o neoplasia subyacente}

\section{Respuesta a los esteroides sistémicos}

\section{Aumento de la VSG}

Se considera SS cuando están presentes los dos criterios mayores y, al menos, dos de los menores.

dolorosas en pies que ascienden hasta tronco, cuello y brazos (Fig. 1). Junto a esto aparece fiebre, artralgias de predominio en grandes articulaciones, con artritis de hombros y tobillos, así como exacerbación de su diarrea. Acude al Hospital en Mayo siendo ingresada. Se objetivan epiescleritis, conjuntivitis, anemia ferropénica de $9,3 \mathrm{gr} / \mathrm{dl}$, VSG $97 \mathrm{~mm} / \mathrm{h}, 16.050$ leucocitos $/ \mathrm{mm}^{3}$ con 14.130 neutrófilos, siendo los estudios inmunológicos realizados (ANA, ANCA,...) negativos. La biopsia de piel fue compatible con el diagnóstico de síndrome de Sweet (Fig. 2). La paciente fue tratada con esteroides a dosis antiinflamatorias desapareciendo la fiebre, las artralgias, las lesiones cutáneas y normalizándose las deposiciones. $\mathrm{Al}$ mes de su alta autosuspendió los esteroides sufriendo un rebrote clínico que respondió nuevamente a la reintroducción de los mismos. Revisada posteriormente se encuentra asintomática en pauta esteroidea descendente.

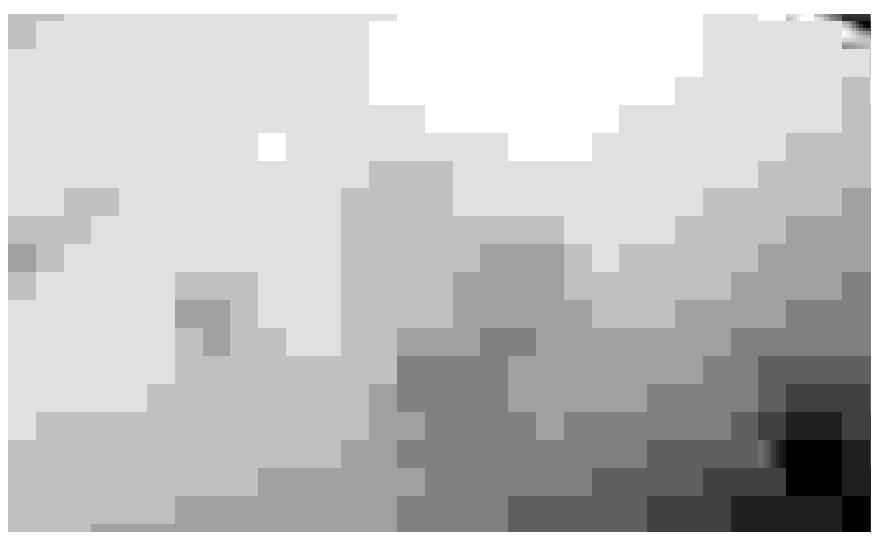

Fig. 1. Lesión eritematosa en espalda.

\section{DISCUSIÓN}

Las dos principales manifestaciones cutáneas de la enfermedad de Crohn (EC) y la colitis ulcerosa (CU) son el eritema nodoso y el pioderma gangrenoso, siendo más frecuente en la EC el primero, y el segundo en la CU (14). El SS es otra manifestación cutánea asociada a la enfermedad inflamatoria intestinal aunque inusual (15). En la literatura se han descrito

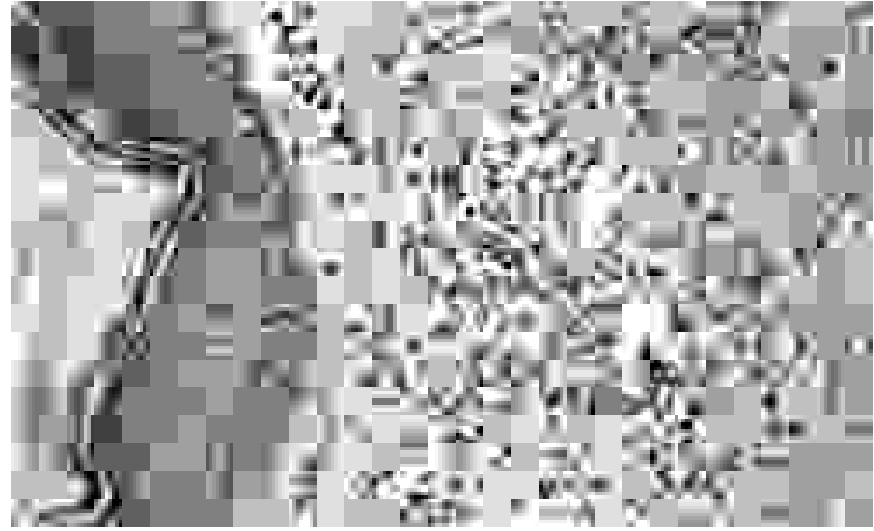

Fig. 2. Biopsia de piel. Se aprecia infiltrado de neutrófilos con edema de dermis (HEx100).

44 casos (15-47) (incluyendo el nuestro) de enfermedad inflamatoria intestinal asociado a SS los cuales se analizan en la tabla II (26 asociado a EC y 18 a CU), en ocho de ellos (6 CU y 2 EC) no se detallan los datos de los pacientes (43-47), por ello no se incluyen en la citada tabla.

El SS se asoció en una proporción superior a EC que a CU (26 casos de EC frente a 18 de CU). El rango de edad osciló entre los 24 años y los 76 años de forma general (rango EC: 25-62; CU: 24-76), con una media global de 46,1 años (media EC: 42,6; CU:53). Existía un claro predominio del sexo femenino, siendo para la EC del $87,5 \%$ y para la CU del $91,6 \%$. El predominio del SS en el sexo femenino es conocido estimándose una proporción $\geq 3: 1(1,40)$, pero cuando se asocia a enfermedad inflamatoria intestinal aún es mayor dicha relación (mujer/hombre: 9:1). La afectación del colon era prácticamente una constante, esto podría justificar el mecanismo patogénico dado que el colon es la principal fuente de bacterias y por ello una potencial fuente antigénica para desencadenar la enfermedad. Generalmente el SS coincidió con un brote de actividad de la enfermedad intestinal sin existir diferencias entre la EC y CU (83,3 y 83\% respectivamente). En un $19 \%$ de los casos el SS precedió entre 1 y 4 años a la aparición de EC, en el 38\% la aparición fue simultánea y en el 42,8\% la EC fue diagnosticada entre 6 meses y 19 años antes de la aparición del SS. Con respecto a la CU en un $75 \%$ fue diagnosticada ésta previamente a la aparición del cuadro dermatológico. La afectación articular fue del $54 \%$ en la EC, manifestándose como artritis franca en el $25 \%$ de los pacientes. En los casos de CU la afectación articular fue ligeramente inferior representando el 33,3\% (25\% con artritis). No existían diferencias con respecto a la frecuencia observada en la enfermedad inflamatoria intestinal no asociada a SS la cual es aproximadamente del $20-25 \%$ (14). Las manifestaciones oculares fueron principalmente conjuntivitis, epiescleritis e iritis, acompañaron a la EC en el $25 \%$ de los casos y a la CU en el $16 \%$. La afectación ocular es más prevalente cuando la enfermedad inflamatoria intestinal se asocia a SS que cuando no lo hace $(3,5 \%$ en la EC y el $10 \%$ en la CU) (14). En la cuarta parte de los pacientes con EC aparecieron otras manifestaciones cutáneas junto al SS, en cinco pacientes se asoció con eritema nodoso y uno presentó un pioderma gangrenoso. En los pacientes con CU esta asociación fue superior, el 41,6\% presentaron pioderma gangrenoso o eritema nodoso acompañado al SS (3 y 2 respectivamente). 
TABLA II

RESUM EN DE LOS HALLAZGOS EN PACIENTES CON SS ASO CIADO A ENFERM EDAD DE CROHN ( EC) Y COLITIS ULCEROSA (CU)

Ell Edad Sexo Distribución Actividad $\quad$ Otroshallazgos $\quad \begin{gathered}\text { Aparición SS en } \\ \text { relación Ell }\end{gathered}$

\begin{tabular}{|c|c|c|c|c|c|c|c|c|}
\hline 1 & $\mathrm{EC}$ & 46 & M & Colónica & Activa & Ninguno & No recogido & 16 \\
\hline 2 & $\mathrm{EC}$ & 25 & M & Colónica & Activa & Iritis, artralgias & 1 año después & 17 \\
\hline 3 & $\mathrm{EC}$ & 52 & M & Colon distal & Activa & Conjuntivitis, artralgias & Simultáneo & 17 \\
\hline 4 & $\mathrm{EC}$ & 57 & $M$ & Colónica & Activa & Conjuntivitis, artritis & Simultáneo & 18 \\
\hline 5 & $\mathrm{EC}$ & 36 & M & Colónica & Activa & Artritis & Simultáneo & 19 \\
\hline 6 & $\mathrm{EC}$ & 23 & M & Colon distal & Activa & $\begin{array}{l}\text { Eritema nodoso, } \\
\text { artralgias, } \\
\text { epiescleritis }\end{array}$ & 15 años después & 20 \\
\hline 7 & $\mathrm{EC}$ & 29 & M & Colon & Activa & $\begin{array}{l}\text { Eritema nodoso, } \\
\text { artralgias }\end{array}$ & 10 años después & 21 \\
\hline 8 & $\mathrm{EC}$ & 54 & $M$ & lleocolónica & Quiescente & Artritis & 1 año antes & 22 \\
\hline 9 & $\mathrm{EC}$ & 33 & M & Colónica & Activa & $\begin{array}{l}\text { Hepatitis crónica activa, } \\
\text { artritis }\end{array}$ & 6 meses después & 23 \\
\hline 10 & $\mathrm{EC}$ & 25 & V & No recogido & Activa & Eritema nodoso & No recogido & 24 \\
\hline 11 & $\mathrm{EC}$ & 39 & $M$ & Perianal & Activa & Artritis & 4 años antes & 25 \\
\hline 12 & $\mathrm{EC}$ & 35 & V & Colónica & Quiescente & Ninguno & 2 años después & 26 \\
\hline 13 & $\mathrm{EC}$ & 45 & $M$ & Ileocolónica & Activa & Artralgias & Simultáneo & 27 \\
\hline 14 & $\mathrm{EC}$ & 47 & $M$ & Colónica & Activa & Ninguno & 19 años después & 28 \\
\hline 15 & $\mathrm{EC}$ & 50 & $M$ & Pancolónica & Activa & Ninguno & Simultáneo & 29 \\
\hline 16 & $\mathrm{EC}$ & 25 & V & Ileocolónica & Activa & $\begin{array}{l}\text { Múltiples abscesos } \\
\text { sistémicos }\end{array}$ & 3 años antes & 30 \\
\hline 17 & $\mathrm{EC}$ & 60 & $M$ & Colon izquierdo & Quiescente & Ninguno & 3 años antes & 15 \\
\hline 18 & $\mathrm{EC}$ & 62 & M & Colon distal & Activa & $\begin{array}{l}\text { Pioderma gangrenoso, } \\
\text { acropaquias }\end{array}$ & Simultáneo & 15 \\
\hline 19 & $\mathrm{EC}$ & 53 & $M$ & Ileocolónica & Quiescente & Conjuntivitis & 11 años después & 15 \\
\hline 20 & EC & 43 & $M$ & Colon izquierdo & Activa & Artritis, poliarteritis & 14 años después & 15 \\
\hline 21 & $\mathrm{EC}$ & 51 & $M$ & Colónica & Activa & Eritema nodoso & 4 años después & 31 \\
\hline 22 & $\mathrm{EC}$ & 49 & M & No recogida & Activa & $\begin{array}{l}\text { Eritema nodoso, } \\
\text { artralgias }\end{array}$ & No recogida & 40 \\
\hline 23 & $\mathrm{EC}$ & 32 & $M$ & Ileocolónica & Activa & Ninguno & Simultáneo & 41 \\
\hline 24 & $\mathrm{EC}$ & 53 & M & Colónica & Activa & $\begin{array}{l}\text { Epiescleritis, } \\
\text { espondilitis } \\
\text { anquilosante }\end{array}$ & Simultáneo & 42 \\
\hline 25 & $\mathrm{CU}$ & 24 & $M$ & Pancolitis & Activa & Pioderma gangrenoso & 3 años después & 32 \\
\hline 26 & $\mathrm{CU}$ & 59 & $M$ & Colon distal & Activa & Artralgias & 14 años después & 33 \\
\hline 27 & $\mathrm{CU}$ & 48 & M & Colon izquierdo & Activa & Ninguno & Simultáneo & 34 \\
\hline 28 & $\mathrm{CU}$ & 76 & M & Colon distal & Activa & $\begin{array}{l}\text { Erupción } \\
\text { vesiculopustular }\end{array}$ & 2 años después & 35 \\
\hline 29 & $\mathrm{CU}$ & 30 & $M$ & No recogido & No recogido & Pioderma gangrenoso & No recogido & 36 \\
\hline 30 & $\mathrm{CU}$ & 42 & $M$ & Subtotal & Quiescente & Sacroileitis & 6 meses antes & 22 \\
\hline 31 & $\mathrm{CU}$ & 58 & $M$ & Colon izquierdo & Activa & Ninguno & 10 años después & 37 \\
\hline 32 & $\mathrm{CU}$ & 40 & V & Colon distal & Quiescente & $\begin{array}{l}\text { Eritema nodoso, } \\
\text { artritis }\end{array}$ & 2 años después & 38 \\
\hline 33 & $\mathrm{CU}$ & 74 & M & No recogido & Activa & $\begin{array}{l}\text { Pioderma gangrenoso, } \\
\text { eritema nodoso }\end{array}$ & No recogido & 24 \\
\hline 34 & $\mathrm{CU}$ & 57 & M & No recogido & Activa & $\begin{array}{l}\text { Conjuntivitis, artritis, } \\
\text { enfermedad hepática }\end{array}$ & No recogido & 24 \\
\hline 35 & $\mathrm{CU}$ & 71 & $M$ & No recogido & Activa & Pioderma gangrenoso & No recogido & 39 \\
\hline 36 & $\mathrm{CU}$ & 58 & M & Colon y recto & Activa & $\begin{array}{l}\text { Epiescleritis, conjuntivitis, } \\
\text { artritis }\end{array}$ & 1 año después & $*$ \\
\hline
\end{tabular}

M mujer. V: varón. Ell: enfermedad inflamatoria intestinal. CU: colitis ulcerosa. EC: enfermedad de Crohn. * Nuestro paciente. 
En resumen, la asociación entre SS y enfermedad inflamatoria intestinal se caracteriza por un claro predominio por el sexo femenino, aproximadamente una relación 9:1, siendo la edad de presentación variable. La afectación del colon ocurre prácticamente de forma constante tanto en la EC como en la CU. Casi siempre coincide con un brote de actividad inflamatoria intestinal, sin existir diferencias entre ambas. Generalmente el SS suele aparecer después del diagnóstico de la CU, siendo la aparición del cuadro cutáneo simultánea o anticipada en el caso de la EC. Las manifestaciones articulares son frecuentes aunque no más que cuando no se asocia a SS. La afectación ocular está presente aproximadamente en la cuarta parte de los casos, una proporción superior a la descrita cuando no se asocia a SS. Existen otras manifestaciones cutáneas acompañantes frecuentemente, fundamentalmente el eritema nodoso y el pioderma gangrenoso.

\section{Bibliografía}

1. Sweet RD. An acute febrile dermatosis. Br J Dermatol 1964; 76: 349-56.

2. Su DWP, Han-Nan HL. Diagnostic criteria for Sweet's syndrome. Cutis 1986; 37: 167-74.

3. Waltz K, Long D, Marks JG, Billinsley EM. Sweet's Syndrome and Erythema Nodosum. Arch Dermatol 1999;135: 62-66.

4. Lear JT, Atherton MT, Byrne JPH. Neutrophilic dermatoses: pyoderma gangrenosum and Sweet's syndrome. Postgrad Med J 1997; 73: 65-8.

5. Mendoza H, Acha V, Vives R, Sánchez J, Berasategui JI, Acero S. Síndrome de Sweet. Presentación de 6 casos y revisión de la literatura. An Med Interna (Madrid) 1997; 14: 244-6.

6. Nguyen KQ, Hurst CG, Pierson DL. Sweet's syndrome and ovarian carcinoma. Cutis 1983; 32: 152-4.

7. Pérez Correa SE, Aladro Benito Y, Suárez Ortega S, Marín Esmenota J, Rivero P, Díaz C. Síndrome de Sweet y afectación de motoneurona asociados a carcinoma de esófago. An Med Interna (Madrid) 1999; 16; 423-6.

8. Schiff BL, Kern AB, Bercovith L. Sweet's syndrome. Report of two atypical cases. Postgrad Med J 1982; 71: 55-60.

9. Prystowsky SD, Fye KH, Goette KD, Daniels TE. Acute febrile neutrophilic dermatosis associated with Sjögren's syndrome. Arch Dermatol 1978; 114: 1234-5.

10. Harray AM. Sweet's syndrome associated with Rheumatoid arthitis. Arch Intern Med 1983; 143: 1993-5.

11. Mizoguchi M, Matsuki K, Mochizuki H. Human leukocyte antigen in Sweet's and its relations hip to Behçets disease. Arch Dermatol 1988; 124: 1069-73.

12. Greer KE, Cooper PH. Sweet's syndrome. Clin Rheum Dis 1982; 8: 427-41.

13. Rodriguez A, Domingo P, Blanc LL, Pérez A, Obrador D. Acute febrile neutrophilic dermatosis associated with postmyocardial infartion syndrome. Arch Intern Med 1985; 145: 1522-3.

14. Levine JB, Lukawski-Trubish D. Extraintestinal considerations in inflammatory bowel disease. Gastroenterol Clin North Am 1995; 24: 633-46.

15. Travis S, Innes N, Davies MG, Daneshmend T, Hughes S. Sweet's syndrome: an unusual cutaneos feature of Crohn's disease or ulcerative colitis. Eur J Gastroenterol Hepatol 1997; 9: 715-20.

16. Sweet RD.An acute febrile neutrophilic dermatosis. $\mathrm{Br} \mathrm{J}$ Dermatol 1964, 76: 349-56.

17. Kemmet D, Gawkrodger DJ, Wilson G, Hunter JAA. Sweet's syndrome in Crohn's disease. BMJ 1988; 297: 1513-4.

18. Villanueva C, Such J, Monés J, Puig LL, Pujol R, Sánchez FJ. Síndrome de Sweet y enfermedad intestinal. Gastroenterol Hepatol 1988; 77: 351-4.

19. Becuwe C, Delaporte E, Colombel JF, Piette F, Cortot A, Bergoend H. Sweet's syndrome associated with Crohn's disease. Acta Dermatovenereol 1989; 69: 444-5.

20. Von den Driesch P, Gómez RS, Kiesewetter F, Hornstein OP. Sweet's syndrome: clinical spectrum and associated conditions. Cutis 1989; 44: 193-200.

21. Schlegel Gómez R, Kiesewetter F, von den Driesch P, Hornstein OP. Sweet-syndrom (akute febrile neutrophile dermatose) und erythema nodosum bei morbus Crohn. Hautarzt 1990; 41: 398-401.

22. Calvo J, González Pérez JA, Febrer I, Oliver V, Herrera A. Síndrome de Sweet. Asociado a enfermedad intestinal inflamatoria crónica. An Med Interna (Madrid) 1990; 7: 364-6.

23. Beitner H, Nakatani T, Hammar H. A case report of acute febrile neutrophilic dermatosis (Sweet's syndrome) and Crohn's disease. Acta Derm Venereol 1991; 71: 360-3.

24. Sitjas D, Puig L, Cuatrecasas M, Moragas JM. Acute febrile neutrophilic dermatosis (Sweet's syndrome). Int J Dermatol 1993; 32: 261-8.
25. Banet DE, McClave SA, Callen JP. Oral metronidazole, an effective treatment for Sweet's syndrome in a patient with associated inflammatory bowel disease. J Rheumatol 1994; 21 : 1766-8.

26. Landi B, Cellier C, Mohib S, Cervoni JP, Barbier JP. Syndrome de Sweet associé á la maladie de Crohn. Presse Med 1995; 24: 39.

27. Ly S, Beylot-Barry M, Beyssac R, Doutre MS, Beylot C. Syndrome de Sweet associé á une maladie de Crohn. Rev Med Intern 1995; 16: 931-3.

28. Burrows NP. Sweet's syndrome in association with Crohn's disease. Clin Exp Dermatol 1995; 20: 279-0.

29. Actis GC, Lagget M, Ciancio A, et al. Recurret Sweet's syndrome in reactivated Crohn's disease. J Clin Gastroenterol 1995; 21: 317-9.

30. Andre M, Aumaitre O, Marcheix JC, Piette JC. Aseptic systemic abscesses preceding diagnosis of Crohn's disease by three years. Dig Dis Sci 1995; 525-7.

31. González Perales JL, Tamarit R, Ballester J, Jiménez Martínez A, Antón MD, Rodriguez Gil FJ, et al. Un caso de síndrome de Sweet asociado a enfermedad de Crohn. Gastroenterol Hepatol 1997; 20: 134-7.

32. Benton EC, Rutherford D, hunter JAA. Sweet's syndrome and pyoderma gangrenosum associated with ulcerative colitis. Acta Derm Venereol 1985; 65: 77-80.

33. Pedreiro J, Jeanmougin M, N’Dyae F, et al. Syndrome de Sweet récidivant, contemporain de poussées de rectocolite hemorragique. Ann Dermatol Venereol 1985; 112: 747-8.

34. Molina M, Ortega G, Pérez B, Pérez A. Síndrome de Sweet y colitis ulcerosa. Rev Esp Enf Ap Dig 1988; 74: 493-4.

35. Villanueva C, Monés J, Pujol R, Puig L, Such J, Sancho FJ. Erupción vesiculopustulosa y síndrome de Sweet asociada a dos exacerbaciones de colitis ulcerosa en una mujer de 76 años. Med Clin (Barc) 1989; 93: 298-300.

36. Kemmet D, Hunter JAA. Sweet's syndrome. A clinicopathologic review of twenty-nine cases. J Am Acad Dermatol 1990; 25: 503-7.

37. Jang HS, Lee JS, Moon DC. A case of acute febrile neutrophilic dermatosis associated with ulcerative colitis. Korean J Dermatol 1991; 29: 522-8.

38. Riesco M, Pardo J, Pérez E, Salaberri J. Síndrome de Sweet con oligoartritis asociado eritema nudoso y colitis ulcerosa. Rev Esp Reumatol 1992; 19: 149-51.

39. Ginarte M, García I, Toribio J. Síndrome de Sweet: estudio de 16 casos Med Clin (Barc) 1997; 109: 588-91.

40. Waltz KM, Long D, Marks JG, Billingsley EM. Sweet's syndrome and erythema nodosum. The simultaneous occurrence of 2 reactive dermatoses. Arch Dermatol 1999; 135: 62-6.

41. Carpels W, Mattelaer C, Geboes K, Coremans G, Tack J. Sweet's syndrome in a patient with Crohn's disease. Acta Gastroenterol Belg 1999; 62 (3): 372-4.

42. Petermann A, Tebbe B, Distler A, Sieper J, Braun J. Sweet's syndrome in a patient with acute Crohn's colitis and longstanding ankylosing spondylitis. Clin Exp Rheumatol 1999; 17 (5): 607-10.

43. Von de Dreisch P. Sweet's syndrome. J Am Acad Dermatol 1994; 31 : 535-56.

44. Fett DL, Gibson LE, Su WPD. Sweet's syndrome: systemic signs and symptoms and associated disorders. Mayo Clin Proc 1995; 70: 234-40.

45. Crow KD, Kerdel-Vegas F, Rook A. Acute febrile neutrophilic dermatosis, Sweet's syndrome. Dermatologica 1969; 139: 123-34.

46. Hommel L, Harms M, Saurat JH. The incidence of Sweet's syndrome in Geneva: a retrospective study of 29 cases. Dermatology 1993; 187: 303-5.

47. Zamora Martínez E, Martín Moreno L, de Castro Torres A, Barat A Síndrome de Sweet. Estudio de diez casos y revisión de la literatura. Rev Clin Esp 1990; 186: 264-9. 